\title{
EFFECTS OF TEMPORAL JUDGMENT ON SOURCE MONITORING AND RESPONSE BIAS
}

\author{
Eriko SUGIMORI ${ }^{1)}$ and Takashi KUSUMI ${ }^{2)}$ \\ ${ }^{1)}$ University of Tokyo, Japan, ${ }^{2)}$ Kyoto University, Japan
}

\begin{abstract}
Our goal was to investigate the effects of temporal judgment on source monitoring and response bias for imagined and perceived drawings. We separated the learning sessions into two days (Day 1 and Day 2) and collected the Day 2 source of each item. The results show that among participants who viewed perceived drawings on Day 1 and imagined items on Day 2, the likelihood of attributing the test items to the perceived drawings category increased with increased repetition of the perceived items. However, when an item was perceived in the learning phase, even if items were imagined repeatedly, the "imagined" responses did not interfere with the rate of "perceived" responses. Furthermore, when an "unknown" response category was included, the response bias of "imagined" decreased, but the response bias of "perceived" did not.
\end{abstract}

Key words: source monitoring, memory, response bias, meta memory

Source monitoring comprises the set of processes involved in making attributions regarding the origins of memories, knowledge, and beliefs (e.g., the spatial, temporal, and social contexts of an event and the media and modalities through which it was perceived; Johnson, Hashtroudi, \& Lindsay, 1993). A specific type of source monitoring, called reality monitoring, is constituted by the process of distinguishing between externally and internally generated information (Johnson \& Raye, 1981; Johnson, 1997).

A typical experimental paradigm in reality monitoring consists of two phases: the learning phase and the monitoring phase. In the learning phase, several items (e.g., an apple) are either generated from an internal source (the subject imagines a drawing of the item) or perceived from an external source (the subject looks at a drawing of the item). The monitoring phase follows the learning phase; each item is presented in turn and participants are asked to judge whether the item was previously generated from an internal source or perceived from an external source. In the typical experimental paradigm, misattribution of not presented items as "imagined" occurs more frequently than misattribution as "perceived," suggesting that misattribution to the source that is less accessible (Bink, Landau, \& Hicks, 1999) or that needs only partial information to recall (Hicks, Marsh, \& Ritschel, 2002) can occur.

We focused on two questions concerning the misattribution of memory sources. The first question involved the need for addressing the factor of temporal judgment, and the second question, the need for investigating participants' responses directly. These issues

Correspondence concerning this article should be addressed to Eriko Sugimori, Department of Cognitive and Behavioral Science, Graduate School of Arts and Science, University of Tokyo, 3-8-1 Komaba, Meguroku, Tokyo, Japan (e-mail: sugimori@beck.c.u-tokyo.ac.jp). 
are addressed in more detail below. First, in the studies to date, each item was learned from only one source (imagining or perceiving a drawing of the item) in a particular learning session. However, because reality monitoring errors usually occur in routine situations, each item should be both perceived and imagined, day after day, and participants should be asked the source of the item shown on a particular day. For example, in everyday life, we are sometimes asked whether we have met our friend A (whom we often meet or think about meeting) at a particular time. In this case, Cohen (1989) suggested that not only do we have to judge whether we have met or imagined meeting him or her, but we also must conduct a temporal judgment. This is why we separated the learning sessions into 2 days (Day 1 and Day 2) and on Day 2 asked for the source of each item in this study.

Studies about memory that have focused on temporal judgment of past events (e.g., Friedman, 1993; Kemp, 1999) have clearly shown that people are not very good at making temporal judgments. When we make a temporal judgment about past events, with the exception of particular special events (e.g., an anniversary), we estimate the date using other relative events. That is, compared to other similar or relative events, the more accessible event is regarded as more recent. Our results show that when an item has been learned repeatedly previously (Day 1 ) and if its memory has become more accessible, although the item is not repeated later (on Day 2), they would be more likely to be regarded as being learned on Day 2 .

Furthermore, when each item is learned both on Day 1 and Day 2 but learned from different sources, and the item is learned repeatedly on Day 1 and its memory becomes more accessible to the subject than that shown on Day 2, the source of the item on Day 2 would be more likely to be misattributed to the source of the item on Day 1. Previous studies have not investigated whether the source of an item of a particular time is influenced by the source of the item at an earlier time. Memories of imagined and perceived information differ in strength and quality (Johnson \& Raye, 1981; Johnson, 1997). Therefore, imagined information and perceived information generated on Day 1 should have differing effects on a source judgment made on Day 2.

Second, previous studies have concluded that misattribution of new items as "imagined" occurs more frequently than misattribution as "perceived" because when subjects feel that they had learned the item but have no idea about the source, they judge that the item must have been learned from the source that is less accessible (Bink et al., 1999) or that needs only partial information to recall (Hicks et al., 2002). However, it is not yet clear whether participants actually attribute items from an unknown source as "imagined." This is why we included an "unknown" response category in Experiment 2. Taking previous findings into account, we predicted that participants would attribute more imagined items as "unknown."

\section{EXPERIMENT 1}

The aim of Experiment 1 was to investigate the factor of temporal judgment under 
the conditions requiring source monitoring. We separated the learning sessions into 2 days (Day 1 and Day 2) and on Day 2 asked for the source of each item in this study. Items were learned in two ways ${ }^{1}$. The first way was that items were not learned on Day 2 but learned only on Day 1. We predicted that both imagining and perceiving items repeatedly on Day 1 would make the items more accessible, and as a result would be regarded as learned from the Day 1 source. That is, under both the Perceived/Not presented (PN) and the Imagined/Not presented (IN) conditions, the likelihood of attributing an item to the Day 1 source will increase as the number of repetitions on Day 1 increases.

The second way was that items were learned both on Day 1 and Day 2 and each item was learned from a different source between Day 1 and Day 2. Our interest was to determine whether imagining repeatedly on Day 1 becomes more accessible than perceiving only once on Day 2 and whether perceiving repeatedly on Day 1 becomes more accessible than imagining only once on Day 2. We predicted that under the Perceived/ Imagined condition (PI), the likelihood of attributing the Day 2 sources as "perceived" will increase as the number of repetitions on Day 1 increases. Furthermore, under the Imagined/Perceived (IP) condition, participants who imagine an item on Day 1 and view pictures of it on Day 2 will not form a bias, as occurs under the PI condition.

\section{METHOD}

Participants. Seventeen Japanese undergraduates participated in this experiment and were tested individually.

Design. The experiment consisted of a 2 (Day 1 source: imagined or perceived drawings) $\times 3$ (frequency of presentation on Day 1: once, three, or eight times) $\times 2$ (Day 2 source: imagined or perceived drawings) within-subject design.

Materials. The 144 items were selected from Amano and Kondo (1999). These norms contained labels for common objects along with line drawings of the objects (Nishimoto \& Hayashi, 1996). The 144 items were divided into 24 sets. A set containing six items was allotted to each condition (Appendix 1). Day 1 source type, Day 1 frequency, and Day 2 source type were factorially combined and counterbalanced within subjects.

In the learning phase on Day 1, eight sets were shown; each of 18 items was presented from the imagining source zero (not presented), one, three, or eight times, and each of 18 items was presented from the perceiving source zero (not presented), one, three, or eight times. In the learning phase on Day 2, each set on Day 1 was categorized as not presented, imagined, or perceived.

Procedure. This experiment consisted of three phases: a learning phase on Day 1, a learning phase on Day 2, and a test phase on Day 2.

In the prior presentation phase, a fixation point was presented for $1 \mathrm{sec}$, followed by a word for $0.5 \mathrm{sec}$. On Day 1, the word was followed by either a perceiving trial or an imagining trial. In a perceiving trial, a drawing representing the word was displayed for $2 \mathrm{sec}$, and subjects were asked to view the drawing for the entire $2 \mathrm{sec}$. In an imagining trial, a blank slide was displayed for $2 \mathrm{sec}$, and subjects were asked to imagine a drawing representing the word for the entire $2 \mathrm{sec}$. During the 2 -sec showing of the line drawing or the formation of a mental image, we asked participants to rate how long it would take an artist to draw the item.

\footnotetext{
${ }^{1}$ We also could have required subjects to learn items on Day 1 and Day 2 using the same source on each day (Perceived-Perceived, Imagined-Imagined), but we omitted this condition for simplicity and to prevent confusion
} 


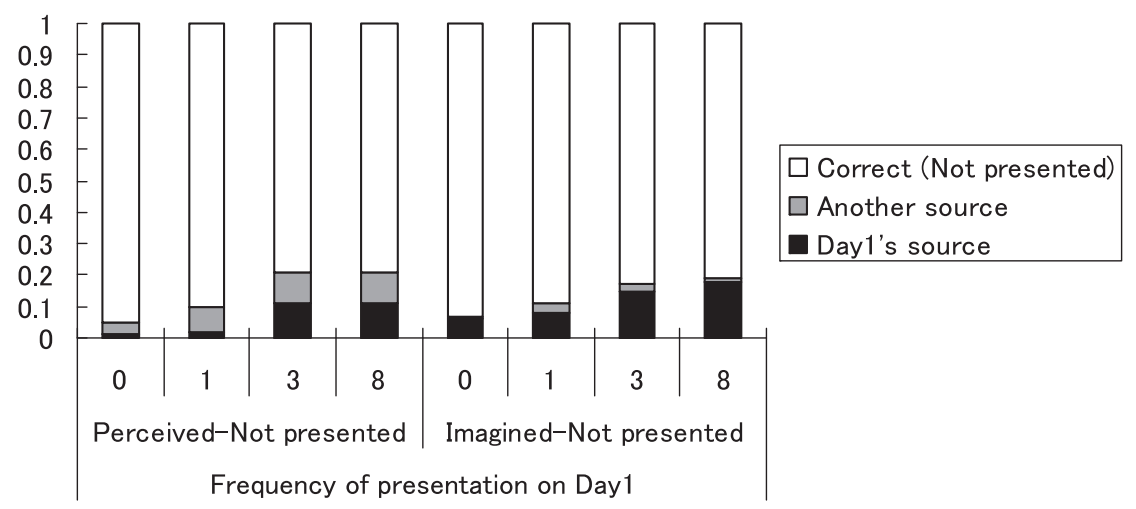

Fig. 1. Mean rate of response to the Day 1 source, another source, or "not presented" (Correct) about the Day 2 source under the condition in which items were not presented on Day 2.

Under the Perceived-Not presented condition (left side), the Day 1 source was "perceived" and another source was "imagined."

Under the Imagined-Not presented condition (right side), the Day 1 source was "imagined" and another source was "perceived."

For each type of trial, perceived or imagined, 54 randomly presented items were included, 18 for each of the three frequencies (i.e., perceiving trials frequencies were referred to as "perceived once," "perceived three times," or "perceived eight times"). In total, the 54 items were presented to each participant 432 times.

The learning phase on Day 2 took place about 24 h later. On Day 2, the 18 items for each condition were categorized as perceived, imagined, or not presented. Each item was presented once, with 96 items in total. The procedures and instruction for the perceiving trials and imagining trials were as described for Day 1. We added the instruction that some items had also been presented on Day 1 and that some of these were generated from a different source.

After the learning phase on Day 2, participants performed a filler task (solving math questions) for 15 min before the source judgment task. In the test phase, 144 items, including items not previously presented, were shown. Participants were asked to specify the source from which they had learned each item on Day 2. We emphasized that they should judge the source of each item from Day 2, and not to choose the Day 1 source. The task was presented on the computer, with each item displayed in the upper half of the screen, and three buttons labeled "Perceived," "Imagined," or "Not presented" were displayed in the lower half.

\section{RESULTS AND DISCUSSION ${ }^{2}$}

Fig. 1 shows the results for the conditions under which items were not learned on Day 2 (PN condition, IN condition). We investigated the factor of temporal judgment simply under the condition in which items were not presented on Day 2; the correct response was "not presented." If participants misattributed the Day 2 source ("not presented") as the Day 1 source (PN condition: "perceived"; IN condition: "imagined"),

\footnotetext{
${ }^{2}$ During preliminary analyses, we checked for differences in judged frequency between drawings that were initially imagined and drawings that were initially perceived. We conducted analyses of variance between initially imagined drawings and initially perceived drawings under each condition, and observed no significant effects under any condition $(p>0.5)$. Consequently, it was not necessary to consider the order effect, and we combined the data.
} 


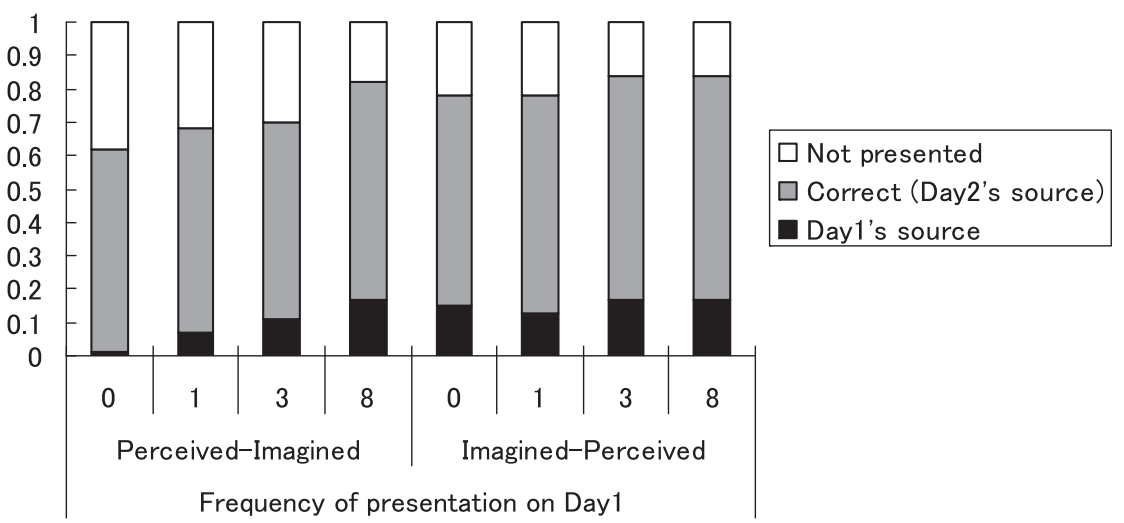

Fig. 2. Mean rate of response to the Day 1 source, Day 2 source, or "not presented" about the Day 2 source under the condition in which items were presented both on Day 1 and on Day 2 and the source was different between Day 1 and Day 2.

Under the Perceived-Imagined condition (left side), the Day 1 source was "perceived" and the Day 2 source (Correct) was "imagined."

Under the Imagined-Perceived condition (right side), the Day 1 source was "imagined" and the Day 2 source (Correct) was "perceived."

the misattribution can be regarded as a temporal judgment error. To investigate whether temporal judgment error (misattribution to the Day 1 source) occurs as the Day 1 presentation increases in both imagined and perceived drawings under Day 1 conditions, we performed analyses of variance (ANOVAs) in which one factor was the Day 1 source type (imagined or perceived drawings) and the second was the Day 1 presentation frequency (zero, once, three, or eight times). With increased repetition on Day 1, even if subjects did not learn the item on Day 2, they were more likely to misattribute the item as a Day 2 source rather than a Day 1 source $(F(3,48)=9.71, M S e=0.01, p<0.01)$.

That is, under both the PN and the IN conditions, although the correct response was "not presented," the likelihood of attributing an item to the Day 1 source increased as the number of repetitions on Day 1 increased and temporal judgment errors occurred. From the viewpoint of the suggestion that temporal judgment is performed by comparing the events' accessibility (Friedman, 1993; Kemp, 1999), we can conclude that repetition of the item makes the item's accessibility higher. Therefore, it was revealed that when an item has been learned repeatedly on Day 1 under both the imagining and perceiving conditions, and its memory has become more accessible, it would likely be regarded as being learned on Day 2 although it was not.

The result that temporal judgment error occurred was also supported by the result that as the number of repetitions on Day 1 increased, the correct response of "not presented" about the Day 2 source decreased $(F(3,48)=9.82, M S e=0.02, p<0.01)$.

Fig. 2 shows the results under the conditions in which items were learned both on Day 1 and on Day 2 and each item was learned from a different source between Day 1 and Day 2 (PI condition, IP condition). If participants misattribute the Day 2 source as the 
Day 1 source (PI condition: "perceived"; IP condition: "imagined"), we can conclude that the Day 1 source became more accessible than the Day 2 source. To investigate whether imagining or perceiving repeatedly on Day 1 becomes more accessible than learning from the other source only once on Day 2, we performed ANOVAs in which one factor was the Day 1 source type (imagined or perceived drawings) and the second was the Day 1 presentation frequency (zero, once, three, or eight times).

A significant interaction was found between the Day 1 source type (imagined or perceived drawings) and the Day 1 presentation frequency (zero, once, three, or eight times) $(F(3,48)=3.01, M S e=0.01 p<0.05)$. That is, under the PI condition, as items were learned from the perceived source repeatedly on Day 1, participants were more likely to regard the items as learned from the perceived source on Day $2(F(3,48)=3.29$, $M S e=0.01, p<0.05)$, although they were in fact learned from the imagined source on Day $2(F(3,48)=1.34, M S e=0.01, n s)$. However, under the IP condition, even though items were learned by being imagined from a source repeatedly on Day 1, participants were not likely to regard the items as learned from the imagined source on Day 2.

From these results, it was revealed that even though items were learned from the imagined source on Day 2, perceiving them repeatedly on Day 1 was more likely to be more accessible than the Day 2 source for participants. Furthermore, temporal judgment errors were likely to occur as items were not learned on Day 2 but learned only on Day 1 (as under the PN and IN conditions). Moreover, it was interesting that when items were learned from an imagined source on Day 1 and learned from a perceived source on Day 2, this kind of error did not occur.

The results that temporal judgment errors occurred under the PI condition and did not occur under the IP condition were also supported by the results that attribution to a not presented source ("not presented") decreased as items were perceived repeatedly on Day 1 (PI condition), and that attribution to a not presented source ("not presented") did not decrease as items were imagined repeatedly on Day 1 (IP condition). Even though the frequency of presentation on Day 1 changed, the learning frequency on Day 2 did not change. Therefore, if the rate of the response to "not presented" changes, it can be said that source monitoring on Day 2 was influenced by the Day 1 learning. These asymmetries support the previous studies suggesting that imagined and perceived sources are different in their strength and quality (Johnson \& Raye, 1981; Johnson, 1997). That is, perceiving repeatedly on Day 1 became more accessible than imagining once on Day 2, while imagining repeatedly on Day 1 did not become more accessible than perceiving once on Day 2.

In addition, we also focused on the response biases under the condition in which items were not learned on Day 1 (See the rate of misattribution to the Day 1 source when the frequency was 0 in Fig. 2). The factor of temporal judgment errors does not have to be taken into account when items were not learned on Day 1. When the Day 2 source was perceived, the source was misattributed to imagined, and when the Day 2 source was imagined, the source was misattributed to perceived. We performed ANOVAs regarding the source misattribution in which the factor was the Day 2 source type (imagined or perceived drawings). As a result, the rate of misattribution to "imagined" was higher than 
that to "perceived" $(F(1,16)=13.31, M S e=0.01, p<0.01)$. This result was the same as in previous studies, suggesting that when participants feel that the item has been learned but they have no idea of the source, they judge that the item must have been learned from the source that is less accessible (Bink et al., 1999) or that needs only partial information to recall (Hicks et al., 2002). In Experiment 2, we include an "unknown" response category and investigated whether participants intentionally attribute unknown items to the source "imagined."

\section{EXPERIMENT 2}

In Experiment 2, our objective was to resolve two questions that arose from the results of Experiment 1. The first was to address how the misattribution rate would change if we added an "unknown" response category. We compared the results of Experiments 1 and 2. If, as previous studies suggest, participants attribute the items' source to "imagined" when they have no idea about the source, the response to "imagined" would be replaced by the response to "unknown."

The second was whether temporal judgment error would not occur under the IP condition even when the frequency of imagined items on Day 1 increases over that of Experiment 1. Thus, we increased the range of presentation frequencies on Day 1, showing each item zero, once, eight, or 15 times. We predicted that under the IP condition, responses in the "imagined" category about the Day 2 source would not change, even if the number of repetitions increased.

\section{METHOD}

Participants. Twenty-one Japanese undergraduates participated in this experiment and were tested individually.

Design. In the prior presentation phase (Day 1), the experiment had three factors: 2 (Day 1 source: imagined or perceived drawings) $\times 3$ (Day 1 presentation frequency: once, eight, or 15 times) $\times 2$ (Day 2 source: imagined or perceived drawings). An additional set of conditions for items that were not presented on Day 1 had the factors 1 (not learned on Day 1) $\times 3$ (Day 2 source: imagined and perceived drawings). Compared to Experiment 1, a greater range of repetition occurred. In addition, we decreased the number of item sets to reduce the overall presentation time.

Materials. In total, 126 items were divided into 21 sets. Each set of six items was allotted to one of the conditions (Appendix 2). Day 1 source type, presentation frequency on Day 1, and Day 2 source type were factorially combined and counterbalanced within subjects.

In the prior presentation phase on Day 1, seven sets were used; in these, 18 items were not presented; 18 items were presented from an imagined source once, three, and eight times; and 18 items were presented from a perceived source once, three, and eight times. In the learning phase, each set from Day 1 was divided into three sets: not presented, imagined, and perceived.

Procedure. The procedure was similar to that described in Experiment 1. However, the item frequencies on Day 1 were different, as previously noted. Eighteen items were presented randomly under each condition; the conditions were referred to as "not presented," "perceived once," "perceived eight times," "perceived 15 times," "imagined once," "imagined eight times," or "imagined 15 times." In total, 864 items were presented to each subject on Day 1. "Not perceived" and "not imagined" in Experiment 1 were 
combined with "not presented" in Experiment 2, reducing the number of conditions in Experiment 2 to seven. On Day 2, 84 items were presented.

In the test phase, 126 items, including new items, were presented. In Experiment 2, the additional response category "unknown" was added, giving participants four response choices, "perceived," "imagined," "not presented," and "unknown." We asked participants to choose the source from which they learned on Day 2. We also emphasized that they must not choose the Day 1 source. We asked participants to choose "unknown" when they remembered that the item had been learned but could not remember the source.

\section{RESULTS AND DISCUSSION}

Fig. 3 shows the results for the conditions under which items were not learned on Day 2 (PN condition, IN condition). Fig. 4 shows the results for the conditions in which items were learned both on Day 1 and on Day 2 and each item was learned from a different source between Day 1 and Day 2 (PI condition, IP condition).

Our first interest in Experiment 2 was to investigate whether participants intentionally attribute the items to "imagined" when they regarded the items as familiar but could not recall the source. We focused on the response biases under the condition in which items were not learned on Day 1 (See the rate of misattribution to the Day 1 source when the frequency on Day 1 was 0 in Fig. 4). When the Day 2 source was perceived, items were misattributed to the imagined source, and when the Day 2 source was imagined, they were misattributed to the perceived source. To investigate how the misattribution rate would change if we added an "unknown" response category, we performed ANOVAs in which the factor was the Day 2 source type (imagined or perceived drawings). As a result, the main effect of the source misattribution was not significant $(F(1,20)=0.25, M S e=0.02, n s)$, that is, the rate of misattribution to "imagined" and that to "perceived" were not different. Compared to the results in Experiment 1 (See the rate of misattribution to the Day 1 source when the frequency on Day 1 was 0 in Fig. 2), in which the rate of misattribution to "imagined" was higher than the misattribution of responding "perceived" under the IN condition, these findings show that the response bias to "imagined" was lower when the response category "unknown" was added. These results support the previous studies' suggestion that items are intentionally attributed to "imagined" when subjects are familiar with them but cannot recall the source.

Our second interest was whether temporal judgment errors would not occur under the IP condition even when frequency of imagined items on Day 1 increased over those of Experiment 1 (Fig. 4). Thus, we increased the range of presentation frequencies on Day 1, showing each item zero, once, eight, or 15 times. If participants misattributed the Day 2 source to the Day 1 source (PI condition: "perceived"; IP condition: "imagined"), we can conclude that the Day 1 source became more accessible than the Day 2 source. To investigate whether imagining or perceiving repeatedly on Day 1 becomes more accessible than learning from the other source only once on Day 2, we performed ANOVAs in which one factor was the Day 1 source type (imagined or perceived drawings) and the second was the Day 1 presentation frequency (zero, once, three, or eight times). A significant interaction was detected between the Day 1 source type (imagined or 


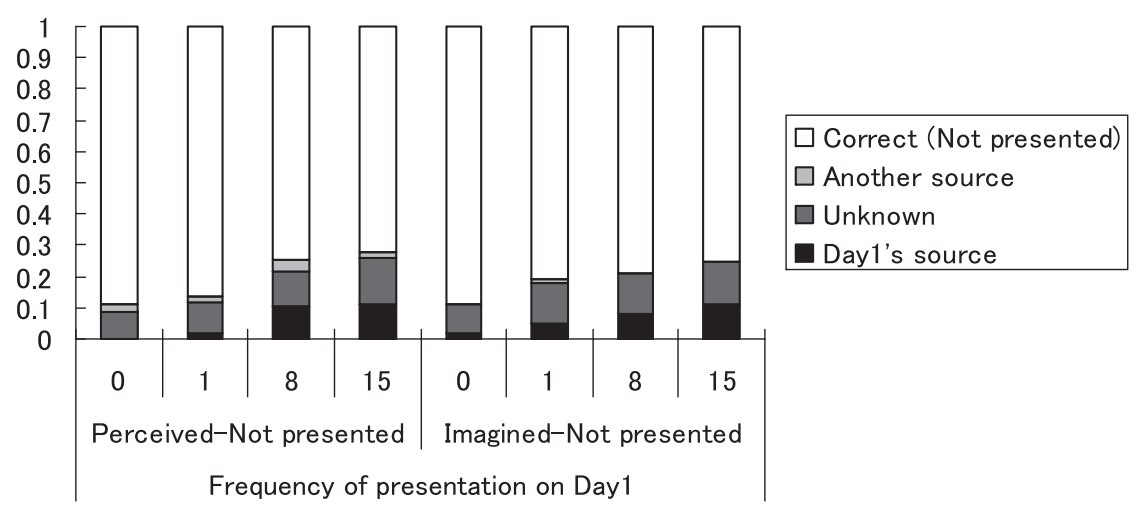

Fig. 3. Mean rate of response to the Day 1 source, "unknown," another source, or "not presented" (Correct) about the Day 2 source under the condition in which items were not presented on Day 2.

Under the Perceived-Not presented condition (left side), the Day 1 source was "perceived" and another source was "imagined."

Under the Imagined-Not presented condition (right side), the Day 1 source was "imagined" and another source was "perceived."

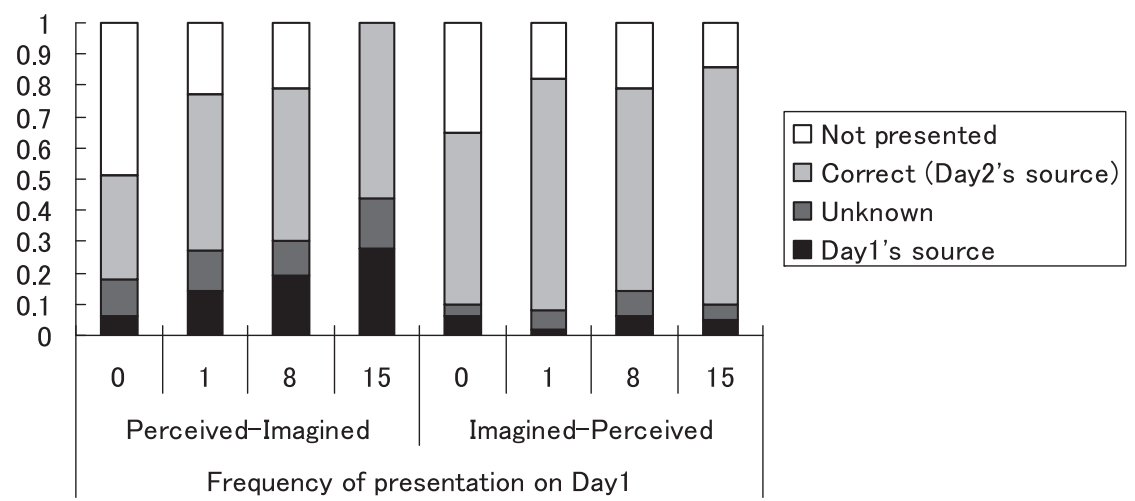

Fig. 4. Mean rate of response to the Day 1 source, "unknown," Day 2 source (Correct), or "not presented" about the Day 2 source under the condition in which items were presented both on Day 1 and on Day 2 and the source was different between Day 1 and Day 2.

Under the Perceived-Imagined condition (left side), the Day 1 source was "perceived" and Day 2 source (Correct) was "imagined."

Under the Imagined-Perceived condition (right side), the Day 1 source was "imagined" and the Day 2 source (Correct) was "perceived."

perceived drawings) and the Day 1 presentation frequency (once, three, or eight times) $(F(3,60)=6.43, M S e=0.01, p<0.01)$. That is, under the PI condition, as items were learned from perceived sources repeatedly on Day 1, participants were more likely to regard the items as learned from a perceived source on Day 2, although they were in fact learned from an imagined source on Day $2(F(3,60)=7.47, M S e=0.01, p<0.01)$. Under the IP condition, however, even though items were learned from an imagined source 
repeatedly on Day 1, participants were not likely to regard the items as learned from an imagined source on Day $2(F(3,60)=0.99, M S e=0.01, n s)$. These results support the conclusion that in Experiment 1, temporal judgment errors occurred under the PI condition and did not occur under the IP condition.

As in Experiment 1, we investigated the factor of temporal judgment under the condition in which items were not presented on Day 2; the correct response was "not presented" (Fig. 3). To investigate whether temporal judgment error (misattribution to the Day 1 source) occurred as in Experiment 1, even though the response category "unknown" had been added, we performed ANOVAs in which one factor was the Day 1 source type (imagined or perceived drawings) and the second was the Day 1 presentation frequency (zero, once, three, or eight times). As the number of repetitions on Day 1 increased, although participants did not learn the item on Day 2, they were more likely to misattribute the Day 2 source as the Day 1 source $(F(3,60)=9.36, M S e=0.01, p<0.01)$. These results support those of Experiment 1.

\section{GENERAL DisCuSSION}

Previous studies have found that misattribution as "imagined" occurs more frequently than misattribution as "perceived." However, in everyday life, misattribution as "perceived" can also occur (e.g., you think you saw friend A today, even though you did not). In this study, misattribution as "perceived" became more frequent when an item was perceived repeatedly previously (Day 1) and when subjects were asked about the Day 2 source even though the item was imagined on Day 2. However, misattribution as "imagined" did not change as the frequency of imagining increased at a previous time (Day 1) and subjects were asked about the Day 2 source ("perceived"). Our study shows the asymmetry between perceived and imagined sources, supporting the theory that memories of imagined and perceived information differ in strength and quality (Johnson \& Raye, 1981; Johnson, 1997). Friedman (1993) suggested that more accessible events are regarded as more recent. That is, perceiving repeatedly on Day 1 became more accessible than imagining once on Day 2.

Furthermore, when we included an "unknown" response category in Experiment 2, misattribution as "imagined" decreased while misattribution as "perceived" did not change. This asymmetry also relates to a difference in accessibility. Participants misattribute a source as "imagined" because imagining is less accessible than perceiving, and participants intentionally attribute a source as "imagined" when they regard the item as old but have no idea about the source. Moreover, participants misattribute a source as "perceived" because perceiving is more accessible than imagining, and temporal judgment errors occur.

We will now discuss future research. In this study, under the PI condition, because items were perceived earlier than they were imagined, imagining items became similar to perceiving on Day 1 and participants regarded the Day 2 source as "perceived." Under the IP condition, because items were imagined earlier than perceived, more differences exist 
between imagining and perceiving, and it may become easier to distinguish the Day 1 source from the Day 2 source. The implications of these findings point to future directions for research employing the source monitoring paradigm.

\section{REFERENCES}

Amano, N., \& Kondo, K. 1999. Characteristics of Japanese vocabulary: Word familiarity (vol. 1). Japan: Sanseido.

Bink, R. L., Landau, J. D., \& Hicks, J. L. 1999. An alternative conceptualization to memory "strength" in reality monitoring. Journal of Experimental Psychology: Learning, Memory, and Cognition, 25, 804809.

Cohen, G. 1989. Memory in the real world. Hillsdale, NJ: Lawrence Erlbaum Associates.

Friedman, W. J. 1993. Memory for the time of past events. Psychological Bulletin, 113, 44-66.

Hicks, J. L., Marsh, R. L., \& Ritschel, L. 2002. The role of recollection and partial information in source monitoring. Journal of Experimental Psychology: Learning, Memory, and Cognition, 28, 503-508.

Johnson, M. K. 1997. Identifying the origin of mental experience. In M. S. Myslobodsky (Ed.), The mythomanias: The nature of deception and self-deception, (pp. 133-180). Mahwah, NJ: Lawrence Erlbaum Associates.

Johnson, M. K., Hashtroudi, S., \& Lindsay, D. S. 1993. Source monitoring. Psychological Bulletin, 114, 328.

Johnson, M. K., \& Raye, C. L. 1981. Reality monitoring. Psychological Review, 88, 67-85.

Kemp, S. 1999. An associative theory of estimating past dates and past prices. Psychonomic Bulletin \& Review, 6, 41-56.

Nishimoto, T., \& Hayashi, S. 1996. The standardization of the picture stimulus for memory experiments. Psychological Reports of Waseda University, 28, 59-85.

(Manuscript received February 21, 2006; Revision accepted October 5, 2007)

Appendix 1. The number of items allotted to each condition, (Experiment 1)

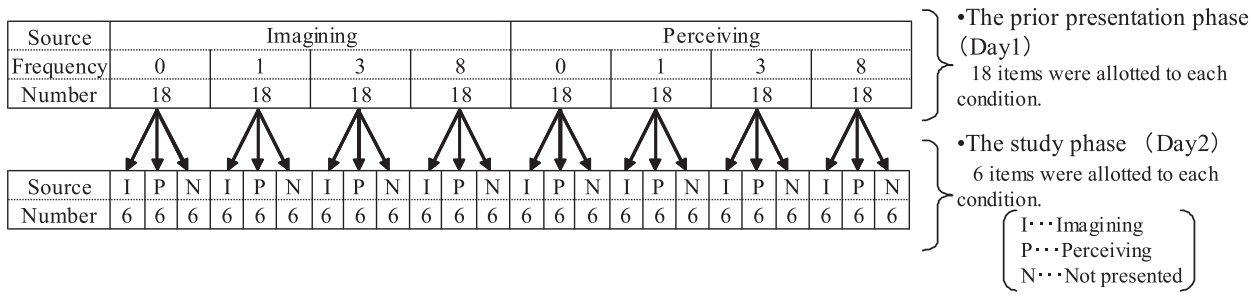

Appendix 2. The number of items allotted to each condition, (Experiment 2)

\begin{tabular}{|c|c|c|c|c|c|c|c|c|c|c|c|c|c|c|c|c|c|c|c|c|c|}
\hline Source & & & & & & & Imagir & ing & & & & & & & Perc & eivi & & & & & - The prior presentation pha \\
\hline Frequency & & 0 & & & 1 & & 3 & & & 8 & & & 1 & & & 3 & & & 8 & & 18 items were allotted to each \\
\hline Number & & 18 & & & 18 & & 8 & & & 18 & & & 18 & & & 18 & & & 18 & & condition. \\
\hline Source & I & $P$ & $\mathrm{~N}$ & I & $\mathrm{P}$ & $\mathrm{N}$ & $\mathrm{P}$ & $\mathrm{N}$ & I & $\mathrm{P}$ & $\mathrm{N}$ & I & $\mathrm{P}$ & $\mathrm{N}$ & I & $\mathrm{P}$ & $\mathrm{N}$ & I & $\mathrm{P}$ & $\mathrm{N}$ & 6 items were allotted $t$ \\
\hline Number & 6 & 6 & 6 & 6 & 6 & 6 & \begin{tabular}{l|l|}
6 & 6 \\
\end{tabular} & 6 & 6 & 6 & 6 & 6 & 6 & 6 & 6 & 6 & 6 & 6 & 6 & 6 & condition. $\mathrm{I} \cdot$. Imagining \\
\hline
\end{tabular}

